\title{
Carbon Footprint Reduction based Smart Path Selection and Data Transmission Practices
}

\author{
Mamta Gupta \\ Department of ECE, \\ CTIEMT, Jalandhar
}

\author{
Harjit Pal Singh \\ Department of ECE, \\ CTIEMT,Jalandhar
}

\author{
Monica Sharma \\ Department of CSE, \\ GNA University
}

\author{
Kritika \\ Department of ECE, \\ Mehar Chand College \\ Jalandhar
}

\begin{abstract}
The carbon footprints define the release of the carbon dioxide in the environment in the wake of the enormous practices. The networking devices consume the larger amount of the energy, which can be significantly reduced by using the energy efficient mechanisms. The carbon emission is responsible for the green house impact, which is the primary reason for the rise in the temperature on the earth. In this paper, the new model for the smarter routing has been proposed for the propagation of the data among the wireless networks. The proposed model has been specifically designed for the energy efficient practices for the reduction of the carbon emission from the wireless networks which involves the wireless node and the wireless BTS. The proposed model has been evaluated under the variety of the experiments, where the proposed model has been found efficient in the terms of the network parameters. The proposed model results have described the proposed model has the most efficient than the existing model as per described in the results section.
\end{abstract}

\section{Keywords}

Carbon emission reduction, Carbon Footprints, $\mathrm{CO}_{2}$, Energy efficiency.

\section{INTRODUCTION}

Greenhouse gases (GHG) are gases in the atmosphere that absorb and emit radiation within the thermal infrared range. These gases prevent heat from escaping from the atmosphere and make the earth warmer. This process is the fundamental cause of the greenhouse effect. The main greenhouse gases in the earth's atmosphere are water vapor, carbon dioxide, methane, nitrous oxide, ozone and chlorofluorocarbons. Human activities add significantly to the level of naturally occurring GHG. Some gases such as hydrofluorocarbons (HFCs), perfluorocarbons (PFCs), and sulfur hexafluoride (SF6) result exclusively from human industrial processes. Greenhouse gases vary in their ability to absorb and hold heat in the atmosphere. HFCs and PFCs are the most heatabsorbent. Nitrous oxide absorbs 270 times more heat per molecule than carbon dioxide, and methane absorbs 21 times more heat per molecule than carbon dioxide.

Mobile subscriber base crossed 5 billion mark in July 2010 and expected to cross 8 billion by $2020^{4}$. With increasing demand for telecom services, the energy consumption has also grown significantly and poses an environment challenge in terms of larger carbon emission footprint of the telecommunication industry. The total global carbon footprint of the ICT industry as a whole is in the order of 860 million tonnes $\mathrm{CO} 2$ which is approximately two percent $2 \%$ of the global emissions. Of this, the contribution from global telecommunication systems-mobile, fixed and communication devices are around 230 million tons $\mathrm{CO} 2$ or approximately $0.7 \%$ of global emissions.

\section{LITERATURE REVIEW}

Kumar, Amit et. al. has worked on the life cycle assessment of wireless bts to reduce carbon footprints. In order to analyze and minimize the impact on environment from whole activities (initial to final process), Life cycle assessment (LCA) should be used which will help in achieving carbon credits. Carbon Credits provide an efficient mechanism to reduce the greenhouse gas emissions by monetizing the reduction in emissions. Rambabu A. Vatti et. al. has described the throughput improvement of randomly deployed wireless personal area networks. In this paper, the authors have proposed a solution to solve the problem of packet loss due to over usage of the intermediate nodes. The authors have proposed a routing algorithm based on the remaining energy at the intermediate nodes. Krishnan, S. S. et. al. has worked on the energy consumption and CO 2 emissions by the Indian mobile telecom industry. The Indian mobile telecom industry, one of the fastest growing sectors in India, had 584.3 million subscribers in 2010-2011 registering an annual growth rate of $49.15 \%$. The energy consumed by the sector was $163 \mathrm{PJ}$ and the corresponding $\mathrm{CO} 2$ emission was estimated at 32.9 million tons. In this paper, the mobile telecom industry is disaggregated into various segments, based on the lifecycle of the device, and each segment's contribution to the overall energy consumption, and its respective $\mathrm{CO} 2$ emissions are discussed. Amanna, Ashwin et. al. has worked on the green communications. The developing world has turned to wireless communications as a leap frog technology past wired communications which spurs its growth even more. Simultaneously, the industrialized world has developed an insatiable demand for broadband data delivered through their cellular handset. This meteoric rise in users and data demand alone does not create a crisis; however when one evaluates the communications ecosystem from a carbon footprint and energy cost perspective, the results are startling.

\section{EXPERIMENTAL DESIGN}

The proposed model has been designed in the three major datasets. The proposed model is designed to analyze the link integrity and propagate the data towards the other nodes. The proposed model components can be enlisted in the following steps:

$\begin{array}{ll}\text { i. } & \text { Pushback Mechanism } \\ \text { ii. } & \text { Carbon Emission Estimation }\end{array}$

\subsection{Pushback Mechanism}

The pushback mechanism has been defined for the link health estimation by assessing the received traffic rate, data loss rate and the carbon emission estimation. The two nodes develop the relationship between them for the data communication in order to evaluate the data rate and the health assessment. The following sequence describes the relationship between the two nodes: 
i. Symbol A describes eh availability and the probability of the node, when it is up and working.

ii. Symbol F describes the node failure probability and depicts the system status as down.

iii. Symbol a describes the node availability

iv. Symbol $\mathrm{n}$ gives the total number of nodes (n) in the network

v. Symbol $\mathrm{s}$ describes the total number $\mathrm{f}$ the inactive nodes in the network

vi. Symbol $\mathrm{f}$ gives the possibilities for the system failure.

The node availability and its probability can be clearly defined with the symbol $\mathrm{A}$ and defined with the following statement:

$$
\mathbf{A}=\mathbf{1 - F} \text {. }
$$

For the very first stage, the connection is considered established in the proposed model simulation. The node availability is assessed by calculating all of the above parameters. The primary objective is to compute the overall probability of the failure, which is given by the following equation:

$$
\mathbf{F}=(\mathbf{1 - a})^{2}
$$

Afterwards, the overall link availability is computed with the following equation:

$$
A=1-F=1-(1-a)^{2}
$$

\begin{tabular}{l} 
Algorithm 1: Pushback Algorithm \\
\hline (1) Begin the pushback module over the node $X$ \\
(2) Establish the connection with the node $Y$ \\
(3) Check for the pushback module on node $Y$ \\
(4) If pushback module will be found active then perform \\
the following sequence: \\
(5) Push module on node $X$ requests the node $Y$ for the \\
network availability evaluation \\
a) Evaluate the incoming traffic from the node $Y$ \\
b) Evaluate the data propagation and forwarding \\
capacity of the egress queue \\
c) Assess the drop rate of the target node \\
(6) Return the decision logic
\end{tabular}

\subsection{Carbon Emission Estimation}

The carbon emission estimation plays the vital role in the proposed model for the data rate limiting based data communication in the wireless networks. The proposed model is based upon the data propagation between the nodes in the non-voice and voice models. The data rate reduction is the method which may utilize the real-time packet rate reduction or the data compression intensity. The proposed model estimates the carbon emission, which if rises than the given threshold, the data rate is limiting request of sent over all of the transmitting nodes.

In India, the average annual threshold for the year 2011, has been recorded at 1.7 metric tons per capita as per defined from the web link. The web link refers to the world bank's statistics, which are given over http://data.worldbank.org/indicator/EN.ATM.CO2E.PC. The per day estimation of the carbon emission has been recorded at 0.0047 metric tons per day, 0.00019 metric tons per hour and 0.0000032 metric tons per minute. Hence, the threshold for the carbon emission based evaluation has been set to $3.2 \mathrm{x}$ $10^{-6}$.

To transmit an email of $1 \mathrm{MB}$ over the internet and read for 5 minutes in the series the $20 \mathrm{~g}$ of the carbon emission $\left(\mathrm{CO}_{2}\right)$. The $1 \mathrm{MB}$ data for one minute can be considered as the $4 \mathrm{~g}$ of the carbon emission $\left(\mathrm{CO}_{2}\right)$. These statistics have been obtained from the online source defined over the link http://www.energuide.be/en/questions-answers/do-i-emit-co2when-i-surf-the-internet/69/. The proposed model has been programmed to utilize these statistics for per minute tracking of the $1 \mathrm{MB}$ data rate.

The following algorithmic defines the complete sequence of the algorithms and defines the complete workflow in the explained manner:

\section{Algorithm 2: Carbon Emission Aware Data Rate Control}

(1) The wireless network cluster is started

(2) The wireless nodes to BTS links are established

(3) BTS node completes the link negotiation stage

(4) BTS node start receiving the data from the wireless nodes

(5) The data rate of the nodes is studied

(6) Track the carbon emission under the every minute routine

(7) Compute the carbon emission for the wireless nodes sending the data

(8) If the carbon emission exceeds the limit of $3.2 \times 10^{-6}$

a. Propagate the message to the nodes to reduce the data rate

(9) Otherwise

a. Propagate the data within the normal data range

(10) End the simulation.

\section{RESULT ANALYSIS}

The result evaluation defines the strength of the proposed model in selecting the paths along the given wireless network. The selection of the longer paths or busier paths may increase the energy consumption as well as the carbon emission over the given nodes.

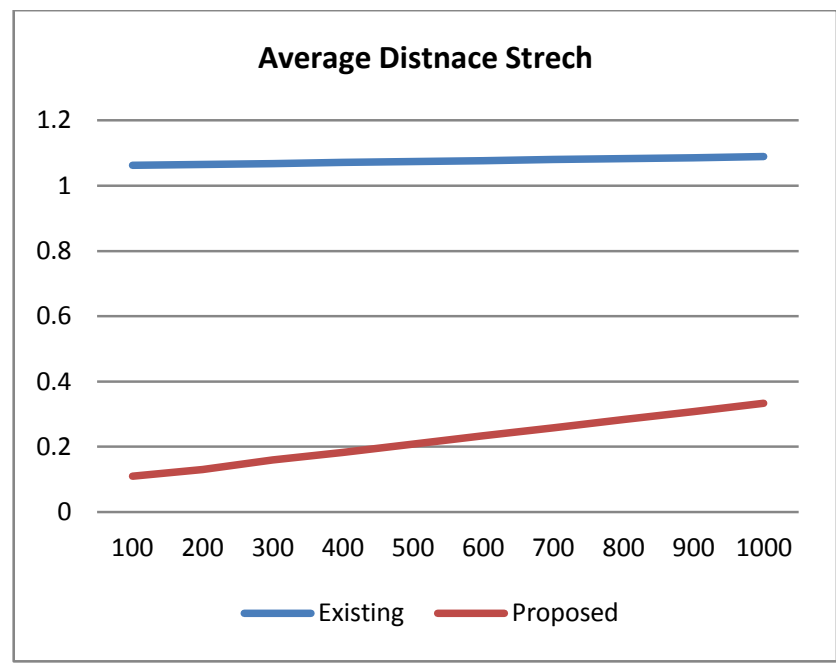

Figure 4.1: The average hop stretch in the proposed path selection model 
The proposed model has been evaluated for the average distance stretch which has been found higher in the case of the existing model, which shows the robustness of the proposed model in electing the comparatively smaller paths. The quality path selection based method in the proposed model has clearly outperformed the existing model, which has eliminated the chances of the higher carbon emission due to the longer path stretches. The following graph (Figure 4.1) describes the robustness of the proposed model in the clear terms also the packet drop ratio has been studied in the proposed model, which gives the another perspective of the network health. The packet drop ratio describes the occupancy of the network resources. The higher occupancy describes the higher data rate, which may also cause the higher energy consumption as well as the higher carbon emission, which must be decreased in order to realize the green wireless network. The carbon footprint reduction has been clearly described from the following figure (Figure 4.2) in the terms of the lower data rate than the existing model. The proposed model results have been described with the series 2 , whereas the series 1 describes the results for the existing model.

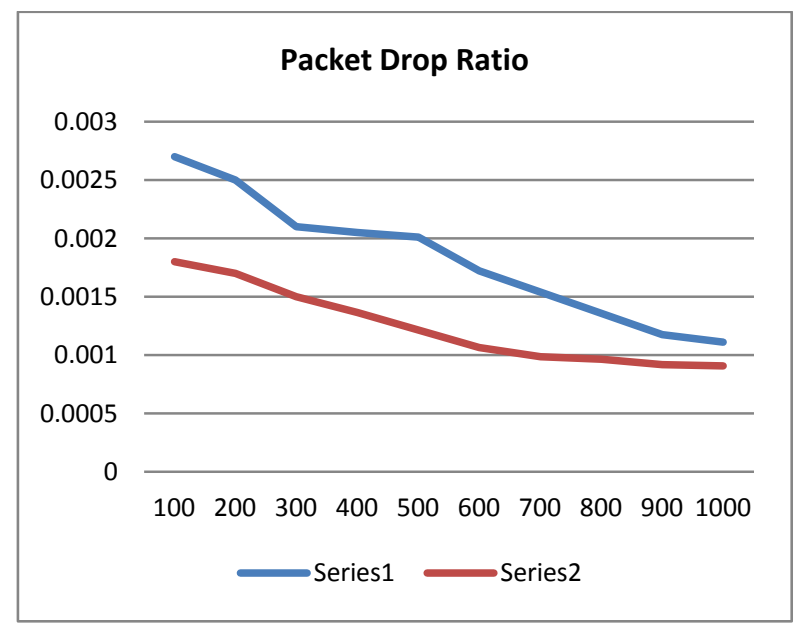

Figure 4.2: The packet drop rate evaluation

\section{CONCLUSION}

The proposed model has been designed with the smart routing with the carbon footprint reduction. The proposed model has been designed with the continuous tracking of the carbon footprint estimation and the data rate reduction. The carbon footprint aware data propagation model describes the data rate for the transmission of the data from the wireless nodes to the wireless base transceiver station (BTS). The wireless BTS is used to control the egress data rate control over the client nodes, which are defined for the carbon footprint control and the overall energy consumption reduction. The proposed model has undergone the various experiments, where the compatibility of the proposed model has been evaluated in detail. The proposed model has been found efficient in comparison with the existing model on the basis of the packet drop rate and the average hop stretch.

\section{REFERENCES}

[1] Kumar, Amit, Tanvir Singh, Rakesh Khanna, and Yunfei Liu. "Life Cycle Assessment of Wireless BTS to reduce Carbon Footprints." In 2013 International Conference on Alternative Energy in Developing Countries and Emerging Economies (2013 AEDCEE), pp. 30-31. 2013.

[2] Rambabu A. Vatti, A.N. Gaikwad "Throughput Improvement of Randomly Deployed Wireless Personal Area Networks, IERI Procedia, Volume 7, pp. 42-48, Elsevier, 2014

[3] Krishnan, S. S., N. Balasubramanian, and A. Murali Ramakrishnan. "Energy consumption and $\mathrm{CO} 2$ emissions by the Indian mobile telecom industry."International Journal of Critical Infrastructures 8, no. 2 (2012): 156-168.

[4] Amanna, Ashwin. "Green Communications." Annotated Literature Review and Research Vision (2010).

[5] Charaan, R. M., R. Ramesh, and N. T. Kumar. "Effective Analysis on R-Leach Protocol for Wireless Sensor Networks." International Journal of Engineering \& Technology (0975-4024) 6, no. 3 (2014). 